\title{
PLAN DE CAPTACIÓN Y MEJORA DE LAS CONDICIONES DE INGRESO DE LAS MUJERES A LAS CARRERAS DE INGENIERÍA DE LA UNIVERSIDAD DE CÓRDOBA
}

\section{KEY STRATEGIES TO ATTRACT AND IMPROVE THE INFLUX OF WOMEN INTO ENGINEERING DEGREES IN THE UNIVERSITY OF CORDOBA}

\author{
Lorenzo Salas Morera (1salas@uco.es) y Rocío Ruiz Bustos (ㅍrbustos@,uco.es)
}

Received: 30/06/2017_Accepted: 20/06/2018

\section{Resumen}

Si bien las carreras de la rama de ingeniería y arquitectura se encuentran entre las que mayor grado de inserción laboral presentan, es un hecho contrastado a nivel nacional e internacional que existe una tremenda descompensación entre el número de mujeres y hombres que eligen este tipo de grados a favor de los últimos; y lo mismo ocurre, aunque en menor proporción, en las titulaciones de la rama de ciencias. Sin embargo, en el conjunto de la Universidad Española, el número de estudiantes mujeres ya ha superado el 50\%. Este hecho sorprendente puede tener diversas causas entre las que se encuentran la mitificación de las ingenierías como "carreras de hombres", la menor (aunque injustificada) confianza de las mujeres en el campo de las matemáticas y la tecnología y, en general, un elevado desconocimiento de los tipos de trabajos que se desarrollan en el campo de la ingeniería.

El presente artículo recoge los resultados tras trabajar con alumnas de institutos y colegios de la provincia de Córdoba en un triple sentido: 1) desmitificar las carreras de ingeniería como poco adecuadas para las mujeres; 2) facilitar toda la información necesaria para una correcta integración en la vida universitaria desde el primer día; y 3) desarrollar actividades académicamente dirigidas que faciliten la transición entre el bachillerato y la universidad.

Palabras clave: Mujeres; Ingeniería; Tecnología.

\section{Abstract}

It is well known that engineering and architect degrees offer real possibilities to insert graduates in the labour market. But, it is also a proven fact that, both at national and international scale, women are a minority in this sector. This also happens in other science degrees, but in smaller percentages.

In the Spanish Universities, more than half of the students are female. This makes the small number of women in technological degrees even more remarkable. Some of the reasons behind these low numbers could be the traditional consideration of degrees "more appropriate for men", the lack of confidence in mathematics and other related science, that women could experience, and in general the tendency of society to discriminate (also in remuneration) women for these jobs. All these, combined with the absence of concrete knowledge of the functions of an engineer, leads to this inequality in the sector.

The idea was to work with high school female students with multiple purposes: 1) change the wrong concept that engineering is more suitable for men, 2) provide accurate information for a right integration of girls in the university before they start their activity on the campus and 3) develop activities addressed to integrated and facilitated the transition between high school and university.

Keywords: Women; Engineering Degrees; Technology. 


\section{INTRODUCCIÓN}

En el año 2015, el número de estudiantes matriculados en las pruebas de acceso a la universidad en todo el territorio nacional ascendió a 287.824, de los cuales el 55\% fueron mujeres y el 45\% fueron hombres. Este ligero desequilibrio a favor de las mujeres en la población que aspira a obtener una plaza como estudiante universitario contrasta sensiblemente con las matriculaciones en las carreras del ámbito de ingeniería. Según datos de la Secretaría General de Coordinación y Seguimiento Universitario del Ministerio de Educación, Cultura y Deporte, para el curso 2014-2015, en todas las áreas de conocimiento es mayor el número de mujeres matriculadas que de hombres, destacando especialmente en este sentido el área de Ciencias de la Salud, con proporciones de $71.18 \%$ para las mujeres y $28.82 \%$ para los hombres; mientras que, por el contrario, en las titulaciones de Ingeniería y Arquitectura, las proporciones se invierten, tomando valores del $25.79 \%$ y $74.21 \%$ respectivamente.

La bibliografía especializada señala como posibles causas fundamentales de este sesgo tan significativo, en primer lugar al desconocimiento de las tareas profesionales propias de la ingeniería y, en segundo, a las metodologías y contenidos propios de las enseñanzas, mientras que el rendimiento académico de las mujeres resulta ser mejor. Sin embargo, y a pesar de este mayor rendimiento, el grado final de inserción laboral de las mujeres es también menor. Con el presente trabajo se pretende incrementar el número de mujeres que eligen carreras de ingeniería en la Universidad de Córdoba así como mejorar su posición a la hora de enfrentarse con el primer curso.

\section{OBJETIVOS}

Como se ha apuntado anteriormente, el objetivo fundamental del trabajo es mejorar el grado de preferencia de las mujeres por las carreras de ingeniería a través de una serie de objetivos secundarios:

1. Identificar los factores que motivan a las mujeres para elegir o descartar carreras de ingeniería.

2. Desmitificar las carreras de ingenería como exclusivas o muy preferentes para hombres.

3. Potenciar las capacidades de las estudiantes de bachillerado y último curso de secundaria para su acceso a titulaciones de la rama de ingeniería.

4. Facilitar la adecuada y no traumática integración en la vida universitaria de las estudiantes en sus primeros años de estudios de ingeniería.

\section{MATERIAL Y MÉTODOS}

En una primera etapa para evaluar el conocimiento, motivaciones y expectativas que tienen las alumnas de secundaria sobre las titulaciones de ingeniería de la Universidad de Córdoba, se han realizado encuestas en los centros que han colaborado con el proyecto (IES La Salle, IES Averroes y IES Colonial, de Fuente Palmera), a alumnas y alumnos de cursos comprendidos entre $3^{\circ}$ de E.S.O y $2^{\circ}$ de Bachillerato.

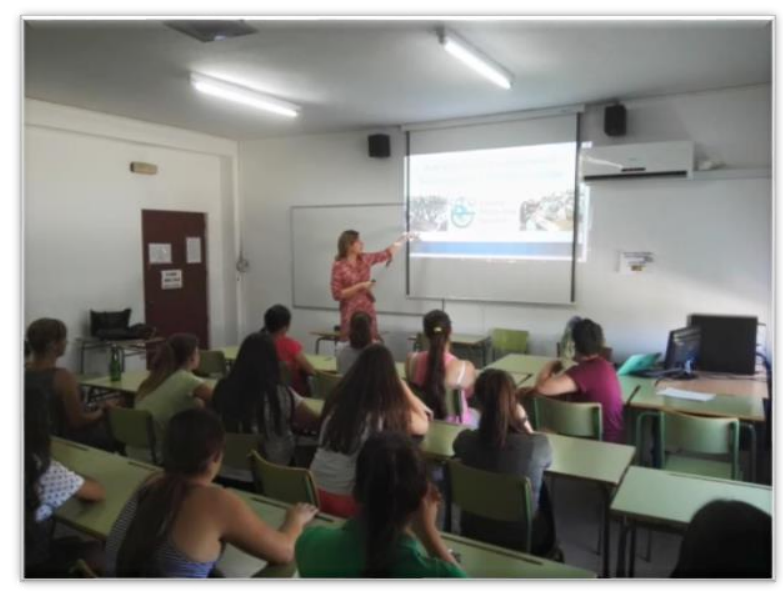

Figura 1.

Impartición de una charla informativa en el IES Averroes. 
Por otro lado, para complementar esta información y obtener una visión global, las encuestas se han hecho extensivas a alumnas de primer curso de ingeniería y también de primer curso de otras titulaciones de ciencias de la UCO. Esto nos va a permitir obtener información muy valiosa, no sólo de las motivaciones que llevan a las alumnas a decantarse por titulaciones ingenieriles, sino también poder conocer la visión y perspectiva de los alumnos que ya se han decantado por otra opción. El número de encuestas es tan elevado, que aún estamos analizando los datos para poder llegar a las conclusiones que nos pueden aportar estos cuestionarios.

En una segunda etapa, se han impartido charlas en los centros de secundaria, en el La Salle y en el IES Averroes (Figura 1), con el objetivo de informar a las chicas de las titulaciones que se imparten en la Escuela Politécnica y resolverles dudas que plantean las alumnas, sobretodo respecto a las futuras salidas profesionales.

\section{RESULTADOS OBTENIDOS Y DISCUSIÓN}

Se organizó un concurso tecnológico con alumnas de los tres centros de enseñanza secundaria involucrados. Se presentaron 21 proyectos en los que hubo 80 participantes de distintos cursos ( $3^{\circ}$ E.S.O., $4^{\circ}$ E.S.O. y $1^{\circ}$ de Bachillerato). La jornada de presentación de los proyectos se celebró el 31 de Mayo en la sala de prensa del Rectorado de la Uninversidad de Córdoba. Durante la misma, las participantes mostraron y defendieron sus proyectos, con ayuda de pósteres.

Los tres grupos finalistas (uno de cada centro) llevaron a cabo una breve presentación oral. También la Ingeniera invitada impartió una charla de mucho interés para las alumnas, en la que les explicó su dilatada carrera profesional en el mundo de la Ingeniería y contestó muchas dudas que las participantes le plantearon. A continuación, el jurado se reunió para deliberar y elegir el proyecto ganador (Figura 2). El jurado estuvo compuesto por la Ingeniera Dña. Ángela Manzano

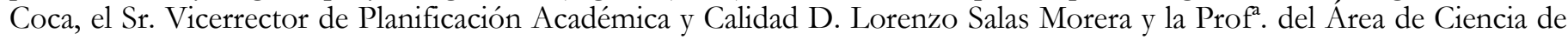
los Materiales e Ingeniería Metalúrgica y Directora del Departamento de Mecánica, Rocío Ruiz Bustos.

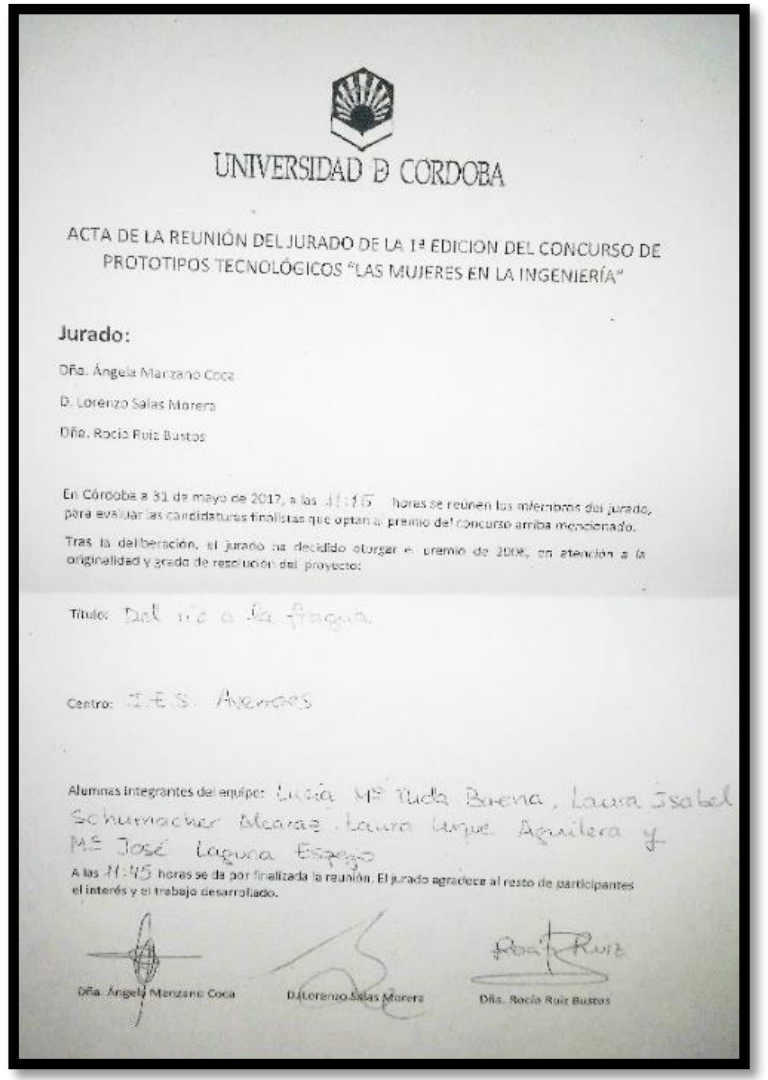

Figura 2.

Acta de la reunión del jurado.
El proyecto ganador fue el presentado por el I.E.S. Averroes (Figura 3). Todas las estudiantes demostrarón ingenio y creatividad con proyectos como un armario capaz de clasificar la ropa con el manejo de una 'app' desde un móvil, un faro de puerto capaz de encenderse mediante la transmisión de poleas o el trabajo ganador del certamen 'Del Río a la Fragua' con el que sus creadoras Lucia María Tuda, María José Laguna, Laura Luque y Laura Schumacher han logrado sustituir el trabajo humano del martillo en la fragua aprovechando la fuerza de la corriente del agua.

El premio, consistente en un cheque de $200 €$, lo entregó el Sr. Rector Magnífico de la Universidad de Córdoba (Figura 4). Durante la celebración del acto animó a las alumnas a interesarse por los distintos grados tecnológicos que oferta la Universidad de Córdoba. 
En la jornada todas las participantes recibieron un diploma de participación y de finalistas y/o ganadoras (Figura 5).

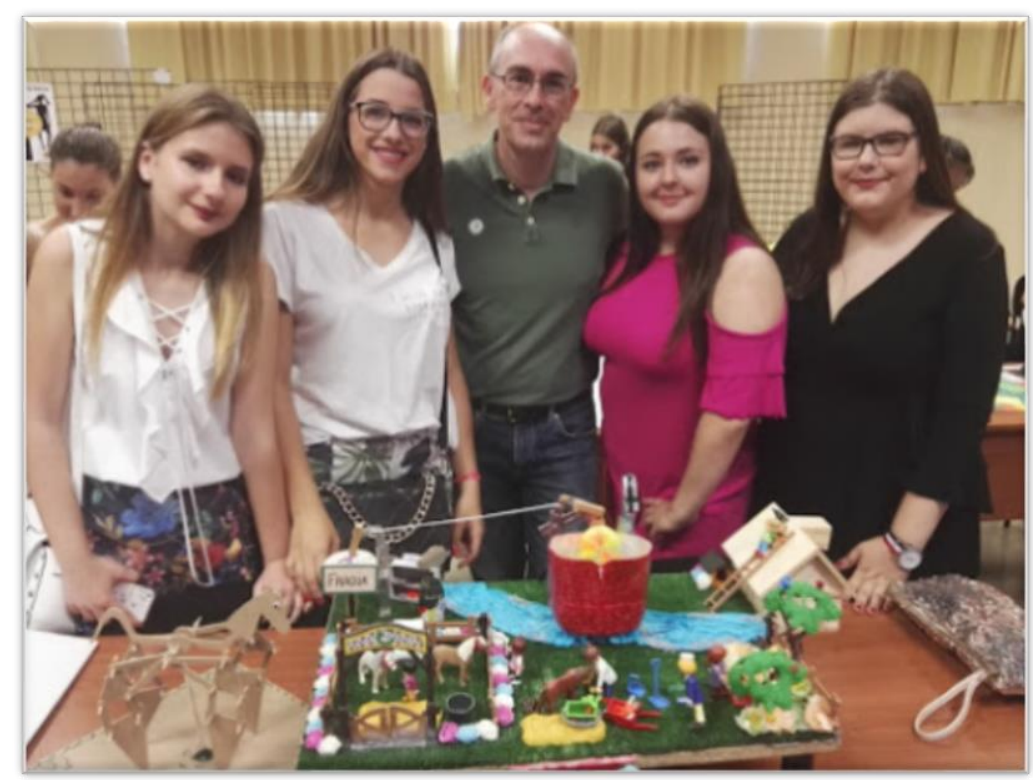

Figura 3.

Equipo ganador del I.E.S. Averroes con el profesor del centro.

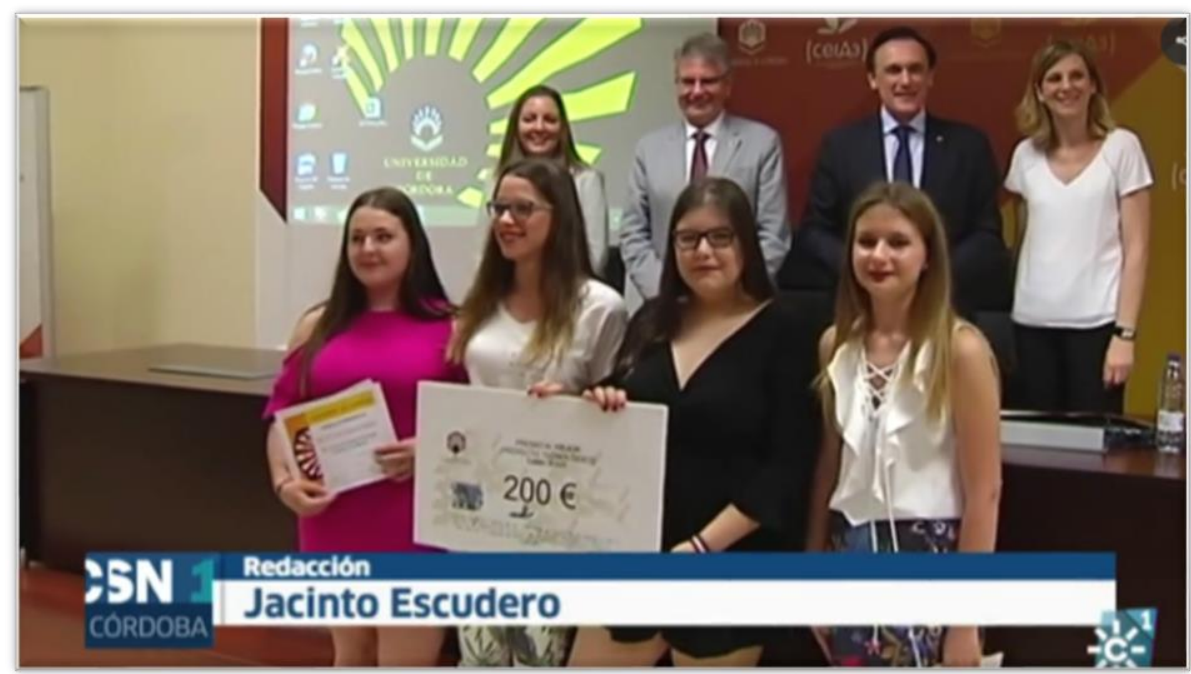

Figura 4.

Entrega del Premio al equipo ganador por el Rector . 


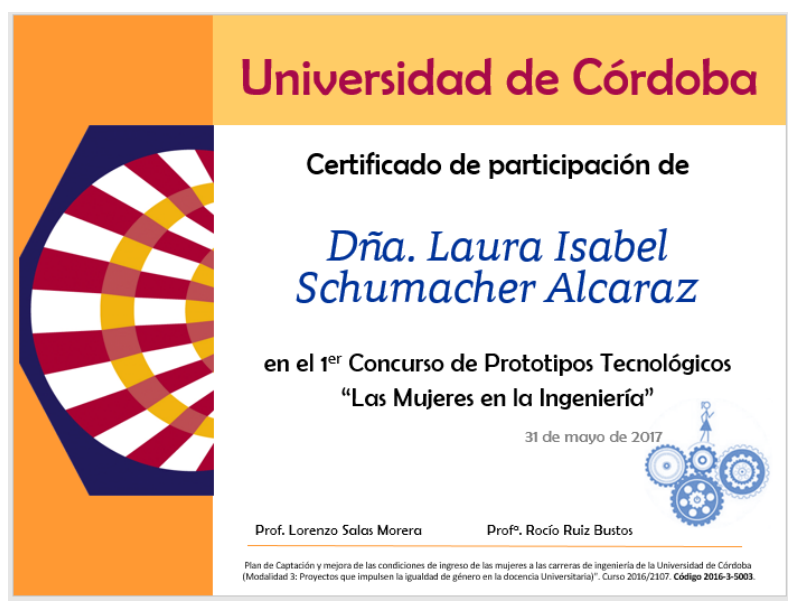

Figura 5.

Ejemplo de uno de los diplomas de participación en el concurso.

\subsection{Resultados obTENidos y Difusión DEL PROYECTO}

La participación ha sido muy buena, con 80 participantes. El análisis de los datos obtenidos en las encuestas, serán muy útiles para poder intentar establecer las causas y posibles soluciones de la problemática que en este artículo se presenta. Por otro lado cabe destacar la amplia difusión que tuvo el concurso en distintos medios, en especial en las noticias de Canal Sur y en el periódico 20 minutos (Figura 6). También en la web de la UCO y del I.E.S. Averroes se publicó la noticia. Esto puede resultar muy importante para que en ediciones sucesivas se animen mas centros a participar y apoyar esta iniciativa.

\section{http://www.juntadeandalucia.es/averroes/centros-tic/14002984/helvia/bitacora/index.cgi?wldPub=2922}

https://www.uco.es/investigacion/ucci/uconews/item/1797-la-uco-distingue-a-un-grupo-de-alumnas-del-institutoaverroes-en-los-premios-las-mujeres-en-la-ingenieria

http://www.canalsur.es/multimedia.html?id=1172859 (Canal Sur Noticias Córdoba, 31/05/2017, de 15:14 a 16:23)

http://www.20minutos.es/noticia/3052286/0/uco-distingue-grupo-alumnas-instituto-averroes-premios-mujeresingenieria

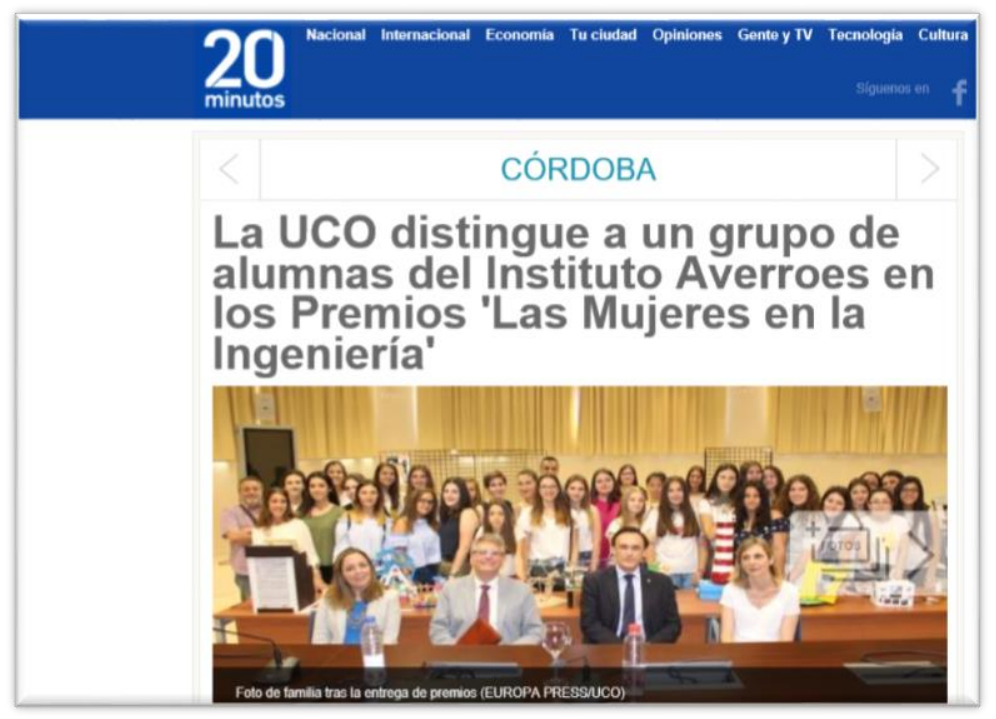

Figura 6.

Publicación del concurso en el medio de difusión “ 20 minutos”. 


\section{CONCLUSIONES}

El interés de las alumnas por los grados ofertados por la Escuela Politécnica Superior de Córdoba ha ido creciendo, según han manifestado los profesores. No obstante, para poder tener resultados mas significativos, es necesario realizar seguir trabajando en esta línea en sucesivos cursos académicos. También debemos conseguir involucrar más a las alumnas de cursos inferiores que aún no han decicido los estudios que van a cursar.

\section{BIBLIOGRAFÍA}

1. Ferrando, F., Paleo, P., De la Flor, S., Urbina, C., \& Gutiérrez-Colon, M. (2012). Estudio sobre la baja presencia de mujeres en los estudios de ingeniería mecánica. In XIX Congreso Nacional de Ingeniería Mecánica (pp. 1-7).

2. Hosaka, M. (2014). Women's experiences in the engineering laboratory in Japan. European Journal of Engineering Education, 39(4), 424-431. https://doi.org/10.1080/03043797.2014.883363

3. Mooney, J. E. (2016). Breaking the stereotype: One female engineer's story. ECN Electronic Component News, 60(8), 12-14. Retrieved from https://www.scopus.com/record/display.uri? eid $=2$-s2.0-84991072051\&origin $=$ resultslist\&sort $=r f \&$

$\mathrm{src}=\mathrm{s} \& \mathrm{st} 1=$ women + engineering $\&$ nlo $=\& \mathrm{nlr}=\& \mathrm{nls}=\& \mathrm{sid}=380 \mathrm{~b} 6 \mathrm{c} 7 \mathrm{ec} 8 \mathrm{deb} 18 \mathrm{e} 1 \mathrm{~b} 5 \mathrm{a} 9481 \mathrm{fb} 391$

$228 \&$ sot $=\mathrm{b} \& \mathrm{sdt}=\mathrm{cl} \& \mathrm{cluster}=$ scopubyr $\% 252 \mathrm{c} \% 25222018 \% 2522 \% 252 \mathrm{ct} \% 252 \mathrm{c}$

$\% 25222017 \% 2522 \% 252 \mathrm{ct} \% 25$

4. OECD. (2015). What Lies Behind Gender Inequality in Education? PISA in Focus (Vol. 49). https://doi.org/10.1787/5js4xffhhc30-en

5. Smith, P., Caputi, P., \& Crittenden, N. (2012). How are women's glass ceiling beliefs related to career success? Career Development International, 17(5), 458-474.

https://doi.org/10.1108/13620431211269702

6. Valle, C., Jackson-Truitt, T., \& Newstetter, W. (2015). How Students Choose Their Engineering

7. Major: Effects of Gender and Race or Ethnicity. In 122nd ASEE Annual Conference and

Exposition, Conference Proceedings (pp. 1-13). https://doi.org/10.18260/p.24197 